\title{
The many factors leading to resurgence of the Blalock shunt for tetralogy
}

\author{
David Zurakowski, PhD, and Richard A. Jonas, MD
}

\begin{abstract}
Feature Editor's Introduction-It is my honor to introduce the expert opinions of Drs Jonas and Fraser, 2 highly gifted and respected surgeons, whom the congenital editors invited to share their not always congruent expert opinions on the surgical management of neonates with tetralogy of Fallot (ToF). Isn't this a "dead horse" issue that had already been pulverized to ash? In a certain sense, yes, but a prestigious cardiology journal recently published a report based on national administrative data that concluded "complete surgical repair for neonates with ToF is associated with a significantly higher risk for early and 2-year mortality compared with the staged approach, after accounting for patient and hospital characteristics." Given what we know about administrative databases, we would likely need to invoke a bit of faith regarding the validity of this specific conclusion. But what was shocking was that the best 2-year survival was only $85 \%$. Really? This is ToF-low single-digit mortality, right? Apparently wrong! This sobering, humbling fact should cause us significant pause. Apparently there is a much bigger horse afoot very much alive and well. So I encourage you to pause, clear your thoughts (and biases), and proceed to enjoy our experts' opinions.
\end{abstract}

\section{Ronald K. Woods, MD, PhD}

In the preprostaglandin years of congenital cardiac surgery during the 1950s, 1960s, and 1970s, neonatal surgical repair carried an unacceptably high mortality and was appropriately avoided in favor of palliative procedures such as shunts and bands. The introduction of prostaglandin E1 allowed newborns with critical heart disease to be stabilized and accurately diagnosed. Ongoing improvements in the hardware of cardiopulmonary bypass such as miniaturization of circuits and technologic improvements in oxygenator design, particularly introduction of membrane oxygenators, opened the door to low-risk major surgical

From the Division of Cardiac Surgery, Children's National Hospital, Washington, DC.

Received for publication Feb 6, 2020; revisions received March 2, 2020; accepted for publication March 5, 2020; available ahead of print June 9, 2020.

Address for reprints: Richard A. Jonas, MD, Division of Cardiac Surgery, Children's National Hospital, 111 Michigan Ave NW, Washington, DC 20010 (E-mail: rjonas@childrensnational.org).

J Thorac Cardiovasc Surg 2021;161:396-9

0022-5223/\$36.00

Copyright (c) 2020 by The American Association for Thoracic Surgery

https://doi.org/10.1016/j.jtcvs.2020.03.172

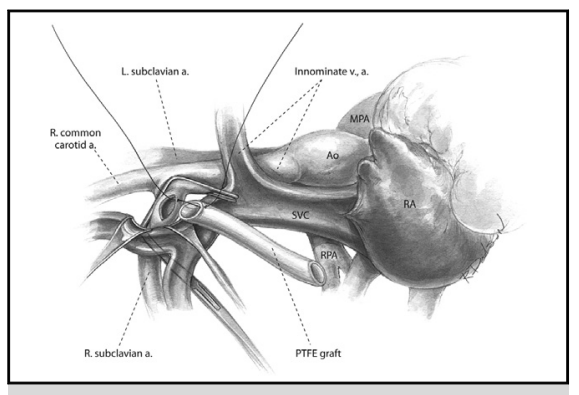

Palliation of tetralogy with a Blalock shunt enhances a surgical program's data analytics.

CENTRAL MESSAGE

Multiple factors are coalescing to reverse the move to early primary repair of tetralogy despite widespread acceptance of neonatal repair of transposition and other symptomatic anomalies.

This Invited Expert Opinion provides a perspective on the following paper: J Am Coll Cardiol. 2019;74(12):1570-1579. https://doi.org/10.1016/j.jacc 2019.05.057.

See Commentaries on pages 400 and 401. 
would choose to palliate a newborn with symptomatic truncus arteriosus or interrupted aortic arch. Even the Norwood procedure, a major neonatal reconstructive procedure, is preferred at most centers over palliative options such as the hybrid approach to hypoplastic left heart syndrome. So an interesting question to ponder is why early repair of tetralogy of Fallot (ToF) has reemerged as a controversy over the last few years, reversing the tide of opinion that was becoming heavily weighted toward early primary repair of ToF 10 to 15 years ago.

\section{GENERAL ADVANTAGES OF EARLY PRIMARY REPAIR}

Early primary repair establishes a normal biventricular circulation that is optimal for the development of all organ systems. ${ }^{3}$ For patients with ToF, as long as the ventricular septal defect is open, pressure in the right ventricle will be systemic and the right ventricle will be progressively more hypertrophied relative to a normal right ventricle. This has important implications in the longer term for diastolic compliance of the right ventricle and the risk of fibrosis and ventricular arrhythmia. The brain is developing rapidly in the first year of life and benefits from normal delivery of oxygen and substrate. A fetal circulation where cerebral oxygenation and blood flow can be reduced is likely to impair brain growth and maturation, so there is catching up to be done. There are clearly important emotional and psychologic benefits for new parents who can be reassured by early repair, thereby removing the stress of a feared repair operation that can be delayed for an indeterminate time if the child undergoes palliation. Finally, several studies have documented the increased cost and increased risk of early mortality with a 2-stage approach including a period of medical management during the interval period. ${ }^{4,5}$

\section{FACTORS DRIVING A REEMERGENCE OF PALLIATION}

\section{Focus on Early Hospital Mortality}

Fortunately, at the time of introduction of the new neonatal arterial switch procedure, Drs Aldo Castaneda and John Kirklin understood the limitations of focusing on early procedural mortality rather than longer-term survival of a diagnostic cohort of patients with transposition. They organized the first multi-institutional study of the Congenital Heart Surgeons Society that used as its end point patient survival by diagnosis rather than early procedural mortality. ${ }^{6}$ Although the alternative Senning and Mustard procedures carried an extremely low procedural mortality as expected, the loss of patients in the preoperative period before a delayed atrial switch more than counterbalanced the slightly higher early mortality of the neonatal arterial switch procedure. In other words, overall patient survival was improved with an operation that carried a higher early mortality. Unfortunately, congenital databases in use around the world today, including the Society of Thoracic Surgeons database used in North America, have focused entirely on early hospital mortality rather than patient survival. The focus on early mortality has been magnified by U.S. News \& World Report program rankings based on Society of Thoracic Surgeons early mortality numbers. There is a misconception that methods of "risk adjustment" can be applied to counterbalance the limitation of early mortality as an end point. This is plain wrong. It is not possible to fully understand the inherent risk of overall mortality without also considering the loss of patients either at the time of the palliative procedure or during the interim period before surgical repair. Of note, the deaths of these patients are simply not included in the early mortality risk after the ultimate repair operation. And the larger denominator resulting from more palliative procedures translates into a lower rate of mortality for the program as a whole.

\section{Impact of Data Analytics}

There are interesting parallels between major sports franchises in the United States and congenital heart programs. A major emphasis today on data analytics has changed decision-making practices in sports where athletes and managers bend the rules or overlook them altogether. Today, hospitals are focused on data analytics including the number of cases performed by surgeons and programs and often provide incentives to promote their own rankings. Shifting from a policy of $100 \%$ early primary repair of ToF to a program of initial palliation below an arbitrary age or weight will substantially increase the number of cases performed by a program, the census of the cardiac intensive care unit, and the relative value units of the surgeons and intensivists on the team.

\section{TEACHING OF NEONATAL SURGERY IN TODAY'S CLIMATE IS DIFFICULT}

An additional consequence of having the microscope of data analytics centered on congenital surgical programs is an increasingly hostile environment for teaching congenital cardiac surgery. Teaching takes time and effort, operating time, and cost, and is not factored into surgical attendings' relative value units or insurance reimbursement. For the last 10 or 15 years, the pipeline of young surgeons graduating with expertise in general cardiothoracic surgery has been drying up, and although the popularity of congenital surgery fortunately remains strong, the applicant pool in the United States is increasingly limited. There is greater dependence on further training in the early years on staff, which is 
hardly conducive to taking on more challenging neonatal repair procedures in one's early years of practice. And there is no question that neonatal repair surgery is more technically challenging and demanding for the surgeon. Nevertheless, a good anatomic result leads to far easier patient management for the cardiac nursing staff and intensive care unit team than palliative alternatives.

\section{RECENT ADMINISTRATIVE DATABASE ANALYSIS OF "TETRALOGY" OUTCOMES}

A review of the recent article by Savla and colleagues, ${ }^{1}$ published in the Journal of the American College of Cardiology, further supports the factors noted, pushing the pendulum back toward more frequent use of palliation for ToF. The article describes an analysis of an administrative database performed at a prestigious institution, the Children's Hospital of Philadelphia. The authors, none of whom is a surgeon, conclude with a powerful statement: "Complete surgical repair for neonates with $\mathrm{ToF}$ is associated with a significantly higher risk for early and 2-year mortality compared with the staged approach, after accounting for patient and hospital characteristics." The persuasiveness of the article is aided by their "Central Image" depicting the weighted survival curves for the first 2 years after the initial procedure. However, the Figure has a $\mathrm{Y}$ axis (probability of survival) that begins just below 0.8 and extends to 1 , thereby magnifying the apparent differences in a visual way between the 2 surgical approaches. There are no confidence bands or error bars to show variability of the estimates, and the curves differ by no more than $5 \%$ at their widest separation.

\section{IS THERE STATISTICAL JUSTIFICATION FOR SUCH A STRONG CONCLUSION BY SAVLA AND COLLEAGUES FAVORING PALLIATION OF TETRALOGY?}

In addition to the well-known limitations of using an administrative database that is maintained primarily for hospital billing, there are a number of other limitations in the article. ${ }^{1}$ Of note, $49 \%(504 / 1032)$ of patients had less than 2-year follow-up in the complete repair group and 36\% (478/1331) in the staged repair group. And if patients with less than 2-year follow-up are excluded, the 2-year mortality rates are $17.4 \%$ in the complete repair group and $17.2 \%$ in the staged repair group. The detailed flow chart in Figure $1^{1}$ shows that patients receiving complete repair had a total mortality of $8.9 \%$ (92/1032) compared with $11.0 \%$ (147/1331) for those undergoing staged repair. The authors ${ }^{1}$ used inverse probability weighting by the propensity score to achieve multivariable adjustment of patient- and hospital-level characteristics. This is an unusual approach given that $k$-nearest neighbor matching based on propensity scores is traditionally used to obtain comparable groups. Therefore, in summary it may not be appropriate to draw such strong conclusions, as those stated in the title and abstract of the article.

\section{WHAT IS THE DEFINITION OF TETRALOGY OF FALLOT IN THE JACC ARTICLE?}

$\mathrm{ToF}$ is a congenital cardiac anomaly that ranges from simple ventricular septal defect physiology ("pink" tetralogy with minimal to no pulmonary stenosis) to pulmonary atresia with hypoplastic pulmonary arteries and multiple collaterals. Nowhere in their article $^{1}$ have the authors from the Children's Hospital of Philadelphia included the term "pulmonary atresia" in the primary article or the previous methodology. ${ }^{7}$ And yet it would seem from the procedural codes applied that their analysis includes patients with tetralogy with pulmonary atresia, presumably many of whom had multiple collateral arteries and hypoplastic pulmonary arteries. Even centers that favor consistent early primary repair rarely recommend elective neonatal repair of ToF. ${ }^{8}$ Thus, the analysis from the Children's Hospital of Philadelphia is looking at the most extreme end of the spectrum and certainly should not be used to imply (as will undoubtedly be inferred by many noting the title of the article alone) that palliation of a previously asymptomatic baby with ToF who is in early infancy should be managed with a palliative approach as opposed to primary repair. However, given multiple other factors pressuring centers and surgeons these days to undertake a 2-stage approach for ToF with pulmonary stenosis, proponents of palliative repair will embrace the strong conclusions of this article with enthusiasm.

\section{AN EVOLVING LANDSCAPE}

The evolving landscape for the training of congenital surgeons has already been described. Another important evolution that is occurring in our field is the increasing percentage of premature and low-birth-weight neonates including many with complex associated anomalies. There are many factors responsible, probably most importantly delayed childbearing and consequent increased use of fertility treatments. Early prenatal diagnosis and the decision by families to continue with a pregnancy despite known anomalies are additional factors. These babies tend to be delivered early because of perceived risks by the management team of continuing a pregnancy in the setting of a known congenital anomaly.

Research studies from 20 and 30 years ago suggested that palliating a baby less than $2.5 \mathrm{~kg}$ by maintaining a 
prostaglandin infusion for several weeks or months until the child had reached an arbitrary larger size resulted in worse outcomes overall than an approach of early primary repair. However, the landscape and surgical practice have been evolving in this area. ${ }^{9,10}$ Although $2.5 \mathrm{~kg}$ was previously considered the tipping point for low birth weight, today a child of that size is considered relatively robust. The question today centers on babies less than 1700 to $1800 \mathrm{~g}$ in whom new and better methods of palliation can be applied, including in the case of symptomatic tetralogy, stenting of the right ventricular outflow tract, and ductal stenting. As interventional catheter techniques for very small babies continue to evolve, there would seem to be a place for a prospective trial of early surgical repair versus palliation. In light of the many factors noted, we can confidently forecast that such a trial will never be done. We hope we are wrong.

\section{Conflict of Interest Statement}

The authors reported no conflicts of interest.

The Journal policy requires editors and reviewers to disclose conflicts of interest and to decline handling or reviewing manuscripts for which they may have a conflict of interest. The editors and reviewers of this article have no conflicts of interest.

\section{References}

1. Savla JJ, Faerber JA, Huang YV, Zaoutis T, Goldmuntz E, Kawut SM, et al. 2year outcomes after complete or staged procedure for tetralogy of Fallot in neonates. J Am Coll Cardiol. 2019;74:1570-9.

2. Castaneda AR, Norwood WI, Jonas RA, Colan S, Sanders S, Lang P. Transposition of the great arteries and intact ventricular septum - anatomic correction in the neonate. Ann Thorac Surg. 1984;38:438-43.

3. Jonas RA. Optimal timing for congenital cardiac surgery: the importance of early repair. In: Comprehensive Surgical Management of Congenital Heart Disease 2nd ed. Boca Raton, FL: Taylor \& Francis; 2014:219-28.

4. Ungerleider RM, Kanter RJ, O'Laughlin M, Bengur AR, Anderson PA, Herlong JR, et al. Effect of repair strategy on hospital cost for infants with tetralogy of Fallot. Ann Surg. 1997;225:779-83.

5. Peer SM, Zurakowski D, Jonas RA, Sinha P. Early primary repair of tetralogy of Fallot does not lead to increased postoperative resource utilization. Ann Thorac Surg. 2014:98:2173-9.

6. Castaneda AR, Trusler GA, Paul MH, Blackstone EH, Kirklin JW. The early results of treatment of simple transposition in the current era. J Thorac Cardiovasc Surg. 1988;95:14-28

7. Savla JJ, Fisher BT, Faerber JA, Huang YV, Mercer-Rosa L. Complete versus staged repair for neonates with tetralogy of Fallot: establishment and validation of a cohort of 2235 patients using detailed surgery sequence review of health care administrative data. Med Care. 2018;56:76-82

8. Cunningham ME, Donofrio MT, Peer SM, Zurakowski D, Jonas RA, Sinha P. Optimal timing for elective early primary repair of tetralogy of Fallot: analysis of intermediate term outcomes. Ann Thorac Surg. 2017; 103:845-52.

9. Bové T, François K, De Groote K, Suys B, De Wolf D, Verhaaren H, et al. Outcome analysis of major cardiac operations in low weight neonates. Ann Thorac Surg. 2004;78:181-7.

10. Chang AC, Hanley FL, Lock JE, Castaneda AR, Wessel DL. Management and outcome of low birth weight neonates with congenital heart disease. J Pediatr. 1994;124:461-6. 